\title{
Characterizing reflections from intraocular lens implants
}

RJ Landry', IK llev', TJ Pfefer', M Wolffe and JJ Alpar

\begin{abstract}
Objective To develop a test method for characterizing glare from intraocular lenses (IOLs) and to confirm a clinical finding that the haptic insertion in the optic of a threepiece IOL produces extraneous line images. Method The method consists of directing a collimated Gaussian laser beam to various parts of the IOL to be tested in a water-filled model eye. Reflected images produced in the retinal plane are photographed with a digital camera.
\end{abstract}

Results A test method was developed to characterize the source of glare images from IOLs. The test method developed was used to confirm a clinical finding that the haptic insertion in the optic of a three-piece IOL produces extraneous line images.

Conclusions The method developed can be used to characterize and pin point the source of extraneous glare images from intraocular lens implants. The haptic insertion in the optic of a three-piece IOL has been identified as a source of line images.

Eye (2007) 21, 1083-1086; doi:10.1038/sj.eye.6702540; published online 25 August 2006

Keywords: IOL glare test method; IOL haptic glare; IOL Line glare image; IOL diffuse glare image

\section{Introduction}

There have been several reports of work to identify the source of unwanted images from intraocular lens (IOLs) implants. ${ }^{1-5}$ Some of the unwanted images appear to the patient as flashes of light, whereas others appear as specific images. In an early paper, Holladay et $a l^{1}$ identified a number of possible sources of mysterious light streaks experienced following extracapsular cataract extraction. They include: car windscreens, spectacle lenses, eye lashes, excessive tear meniscus, intraocular lens scratches, and posterior capsule corrugations. In another paper by Holladay et $a l_{1}^{2}$ ray trace analyses were used to show that reflections from the sharp edge of an IOL produce a sharp crescent image whereas reflections from a round edge produce a diffuse image. The peripheral positioning holes in IOLs with a $4.3 \mathrm{~mm}$ clear optical zone have been reported as the source of many visual complaints $\left(\right.$ Landry $\left.{ }^{3}\right)$. Alpar ${ }^{4}$ also described how the clear cylindrical shaped loops in older iris-clip implants, which are attached at a $90^{\circ}$ angle to the posterior lens body, act as a light pipe. When the lens is illuminated the entire posterior loop also becomes illuminated, thereby causing a disturbing visual phenomenon. Finally, in a recent paper, it was shown that the haptic insertion in a three-piece IOL could be the source of line images arising from point sources of light such as headlamps from oncoming cars. $^{5}$

As there can be several sources of unwanted images following IOL implantation, it is important to have an objective method which can be used to identify the source of extraneous images or glare following cataract surgery. In this paper, we report on a relatively simple laboratory method that was used to simulate the glare images reported by a patient following implantation of a three-piece silicone IOL.

\section{Materials and methods}

Figure 1 is a schematic optical diagram of the experimental set-up. It includes a $15 \mathrm{~mW}$ power, continuous wave, laser diode (Melles Griot Model 561C5153/HS) operating at a wavelength of $658 \mathrm{~nm}$ and a water-filled model eye containing the IOL to be evaluated. A $10 \times$, $16.5 \mathrm{~mm}$ focal length, and objective lens (O1) is used to focus the laser beam down a $1 \mathrm{~m}$ long,
${ }^{1}$ Division of Physics, Center for Devices and Radiological Health, FDA, Rockville, $M D$, USA

${ }^{2}$ Scientific Consultant, Moseley, Birmingham, UK

${ }^{3}$ St Luke Eye Institute, Amarillo, TX, USA

Correspondence:

RJ Landry,

Division of Physics, HFZ-130,

Center for Devices and Radiological Health,

FDA, 9200

Corporate Boulevard, Rockville, MD 20850, USA

Tel: + 1301827 4687;

Fax: + 13018274677

E-mail: robert.landry@

fda.hhs.gov

Received: 27 January 2006 Accepted in revised form:

5 July 2006

Published online: 25 August 2006

Financial/proprietary interest: The authors do not have any commercial or proprietary interest in any of the products referred to in this paper 
$4 \mu \mathrm{m}$ diameter, single mode fibre. This produces a Gaussian beam profile at the output, the position of which coincides with the focal point of a $100 \times, 1.9 \mathrm{~mm}$ focal length, objective lens (O2). The resultant $0.5 \mathrm{~mm}$ diameter collimated Gaussian beam is directed at various angles of incidence to various points on the IOL. The IOL is situated in the water-filled model eye, which in turn, is located on a rotating plate that could be linearly translated from side to side enabling accurate positioning of the laser beam on the IOL.

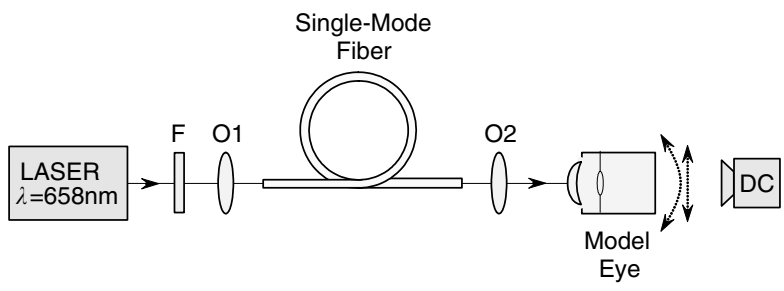

Figure 1 Experimental set-up used to characterize glare from IOLs. The set-up includes: a $15 \mathrm{~mW}$ power, continuous wave, laser diode (Melles Griot Model 561C5153/HS) operating at a wavelength of $658 \mathrm{~nm}$ and a water-filled model eye containing the IOL to be evaluated. F is a transmission filter that provides control of the power of the laser beam. (O1) is a $10 \times, 16.5 \mathrm{~mm}$ focal length, objective lens used to focus the laser beam down a $1 \mathrm{~m}$ long, $4 \mu \mathrm{m}$ diameter, single mode fibre. (O2) is a $100 \times$, $1.9 \mathrm{~mm}$ focal length, objective. The water-filled model eye is located on a rotating plate that can be linearly translated from side to side.
The model eye is fitted with a hard contact lens with a radius of curvature of $7.7 \mathrm{~mm}$. The three-piece silicone IOL used was located in the normal position in the eye with respect to the cornea and at a distance of $17 \mathrm{~mm}$ from the retinal plane. The edge of the IOL is flat with rounded corners. A circular flat glass plate with a diameter of $3 \mathrm{~cm}$ is located in the retinal plane so that images in this plane can easily be viewed by placing vellum over the external part of the glass plate. Images formed on the vellum when the laser beam is directed to various parts of the IOL are photographed with a Canon Power Shot S 60, 5.2 megapixels digital camera (DC). The shutter speed and aperture of the DC are varied until an accurate photographic representation of the image seen visually on the vellum in the retinal plane is obtained.

\section{Results}

Figure 2 (a) is a photograph of the image on the vellum when the laser beam is incident on the haptic outside of the optic of the three-piece IOL. The angle of incidence of the collimated laser beam on the cornea is nearly parallel to the optical axis of the model eye. As can be seen from Figure 2(a), a line image is produced with a bright central spot from the laser beam. For this photograph, the shutter speed was $1 / 50$ th of a second and the aperture was $\mathrm{f} / 4$.

Figure 2(b) is a photograph of the image on the vellum when the laser beam is incident on the haptic within the optic of the IOL. The angle of incidence of the laser beam
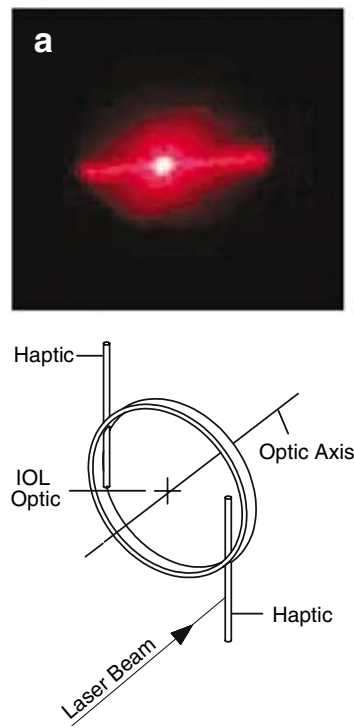
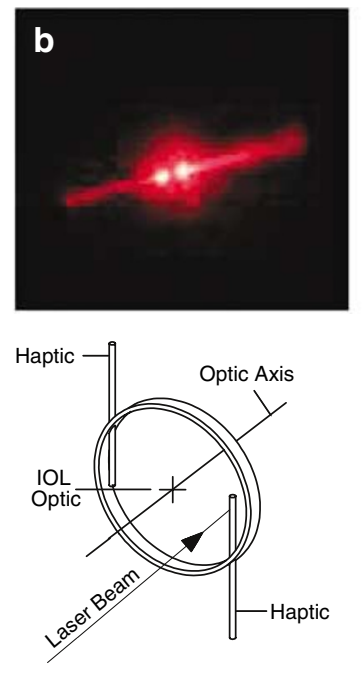
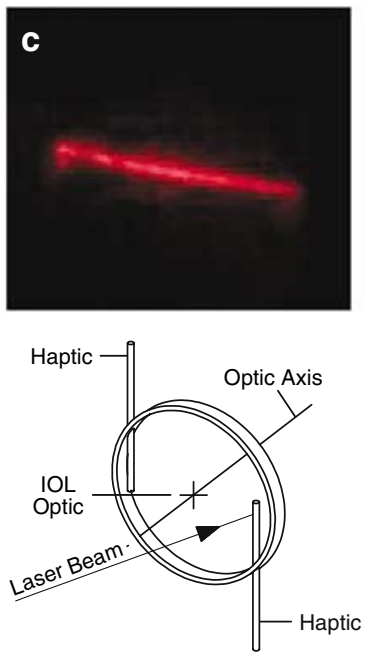
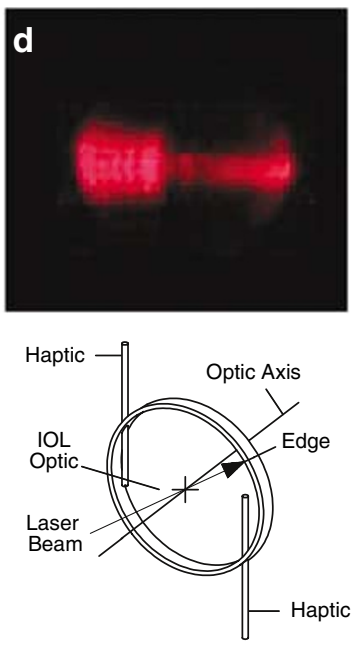

Figure 2 Digital photos of images on vellum when the laser beam is directed to various parts of the IOL at various angles relative to the optical axis along with sketches showing the location and angle of incidence of the laser beam on various parts of the IOL for the laser beam incident on: (a) the haptic outside of the optic of the three-piece IOL at an angle of incidence nearly parallel to the optical axis of the model eye. (b) The haptic within the optic of the IOL at an angle of incidence nearly parallel to the optical axis of the model eye. (c) The haptic within the optic of the IOL at an angle of incidence approximately $33^{\circ}$ relative to the optical axis of the model eye. (d) The edge of the IOL optic at an angle of incidence approximately $30^{\circ}$ relative to the optical axis of the model eye.' 
on the cornea was again nearly parallel to the optical axis of the eye model. As can be seen from Figure 2(b), a line image with two bright central spots from the laser beam is produced. For this photograph, the shutter speed was $1 / 100$ th of a second and the aperture was $f / 2.8$.

Figure 2(c) is a photograph of the image on the vellum when the laser beam is incident on the haptic within the optic of the IOL. In this case, the angle of incidence of the laser beam on the cornea is approximately $33^{\circ}$ relative to the optical axis of the model eye. For this photograph, the shutter speed was $1 \mathrm{~s}$ and the aperture was $\mathrm{f} / 7.1$.

Figure $2(d)$ is a photograph of the image on the vellum when the laser beam is incident on the edge of the IOL optic. The angle of incidence of the beam on the cornea is approximately $30^{\circ}$ relative to the optical axis of the model eye. As can be seen, a dense diffuse image is formed with lines that run from the diffuse image to the source of the glare at the edge of the IOL. For this photograph, the shutter speed was $1 / 10$ th of a second and the aperture was $\mathrm{f} / 2.8$.

\section{Discussion}

Figure 2(a) shows that a line image is produced in the retinal plane when light from a point source strikes a part of the haptic outside of the IOL optic. A line image is consistent with reflections from a cylindrical object in the light path. Figure 2(b) shows that a line image is also produced when the light from the point source strikes the haptic insertion in the IOL optic. In this case, the line image is similar to that produced when the light is incident on the haptic alone and is similar to the line image described by a patient. ${ }^{5}$

It should be noted that for patients who have a $5 \mathrm{~mm}$ diameter mesopic pupil and are fitted with a $6 \mathrm{~mm}$ diameter three-piece IOL, it is highly likely that they may experience distracting line images if the IOL is decentred by as little as $0.5 \mathrm{~mm}$. In such a case, the IOL haptic will be positioned within the patient's pupil. The haptic can thus be directly illuminated from sources, such as automobile headlamps, thereby producing the distracting line images. These symptoms can occur not only when the illuminating angle relative to the optical axis is small, but also when it is as large as $30-80^{\circ}$. This can occur from headlamps of oncoming cars as they pass by or when headlamps from cars following are reflected from side view mirrors. Indeed line glare images have been experienced under such conditions. ${ }^{5}$

Figure 2(c) shows that a line image is also produced when the light from the point source strikes the haptic insertion at an angle of about $33^{\circ}$ relative to the optic axis of the IOL. In this case, the line image is similar to that produced when the light is incident on the haptic alone. It is also similar to the line image described by a patient from the reflections from the side view mirror of a car. ${ }^{5}$

Figure 2(d) shows the image that is produced when the collimated laser beam is directed to the edge of the IOL away from the haptic insertion at angles between $30^{\circ}$ and $40^{\circ}$ relative to the optical axis of the eye. In this case, a diffuse image is produced that extends across the field of view. As can be seen, the image is dense with discernible perpendicular lines on the side of the retina opposite the glare source at one end with straight lines extending from the dense portion of the image to the glare source. This image appears to be similar to the cobweb-like image described by a patient. $^{5}$

Finally, the line images shown in Figure 2(a)-(c) in this paper, which are produced by the haptic of a three-piece IOL, are different from the light effects reported by Landry and Alpar. ${ }^{3,4}$ In the case of the $6 \mathrm{~mm}$ diameter lens used in this study, the clear optical zone is $5 \mathrm{~mm}$ as compared to the $4.3 \mathrm{~mm}$ clear optical zone in the lenses with positioning holes $\left(\right.$ Landry $\left.^{3}\right)$. Further, the patients with lenses containing positioning holes reported seeing 'rings of light'; as opposed to the line images produced by reflections from the haptic insertion and the diffuse images produced by the edge of the IOL. Finally, the line images seen by the patient with the three-piece IOL are certainly different from the light pipe effect reported by Alpar.

\section{Conclusion}

We have shown that the haptic insertion in a three-piece IOL can give rise to extraneous line images. This confirms the clinical finding that the haptic insertion in the optic of a three-piece silicone IOL is the source of symptoms of an unwanted line image. ${ }^{5}$ This finding suggests that, in the case of three-piece IOLs, the IOL haptic should be considered as a possible source of glare images in patients with large mesopic pupils. We have also shown that, depending on the angle of incidence, the edge of an IOL with rounded corners can give rise to a more diffuse extraneous image that extends from the source of the glare image across the entire field of view. Finally, it has been shown that this laboratory test method can be used to accurately pin point the locations of reflections originating within an IOL. Consequently, it can also be used as a means of assessing the likelihood that specific IOL designs might produce unwanted glare images. However, as the method uses a laser beam, it can only be used to pin point the sources of glare resulting from point sources of light such as the headlamps of cars. It cannot be used, for example, to determine glare that may arise from spherical aberration. 


\section{Disclaimer statement}

The mention of commercial products, their sources, or their use in connection with material reported herein is not to be construed as either an actual or implied endorsement of such products by the United States Department of Health and Human Services.

\section{References}

1 Holladay JT, Bishop JE, Lewis JW. Diagnosis and treatment of mysterious light streaks seen by patients following extracapsular cataract extraction. J Am Intraocul Implant Soc 1985; 11: 21-23.
2 Holladay JT, Lang A, Portney V. Analysis of edge glare phenomenon in intraocular lens edge design. J Cataract Refract Surg 1999; 25: 748-752.

3 Landry R. Unwanted optical effects caused by intraocular lens positioning holes. J Cataract Refract Surg 1987; 13: 421-423.

4 Alpar JJ. Glare factor in different intraocular lenses: Comparative study in Patients $\mathrm{w}$ ith dissimilar binocular implants. In: Emery JM and Jacobson AC (eds). Current Concepts in Cataract Surgery, Selected Proceedings of the Seventh Biennial Cataract Surgical Congress. Appleton-Century-Crofts: New York, 1982.

5 Wolffe M, Landry RJ, Alpar JJ. Identification of the Source of Permanent Glare from a Three Piece Silicone IOL. Eye 25 August 2006 [E-pub ahead of print, doi:10.1038/sj.eye.6702539]. 